Case Report

\title{
Thoracoscopic Plication for Diaphragmatic Eventration in a Neonate
}

\author{
Tsubasa Takahashi, MD, ${ }^{1}$ Tadaharu Okazaki, MD, ${ }^{1}$ Takanori Ochi, MD, ${ }^{1}$ \\ Kinya Nishimura, MD, ${ }^{2}$ Geoffrey J Lane, MD, ${ }^{1}$ Eiichi Inada, MD, ${ }^{2}$ and Atsuyuki Yamataka, MD
}

\begin{abstract}
Background: Currently, thoracoscopic surgery is replacing thoracotomy for an ever increasing number of indications, even in pediatric surgery. However, there are few reports describing thoracoscopic plication (TP) for diaphragmatic eventration in children, particularly in neonates. We report a case of TP under single-lung ventilation in a neonate with diaphragmatic eventration.

Case Report: A 10-day-old boy was referred for surgical management of right diaphragmatic eventration. Birth was at term, following an uncomplicated pregnancy and delivery. Shortness of breath, labored respiration and chest retraction presented soon after birth, necessitating mechanical ventilation. Chest radiography and computed tomography revealed an elevated right hemidiaphragm. Attempted weaning off mechanical ventilation failed with persistence of respiratory symptoms, requiring nasal directional positive airway pressure. However, because there was no resolution of symptoms, TP was performed using a 3 port technique under single-lung ventilation on day 17 of life. The postoperative course was excellent with complete resolution of respiratory symptoms with no recurrence for 9 months.

Conclusion: To the best of our knowledge, this is the youngest case of TP for diaphragmatic eventration performed under single-lung ventilation. TP is safe, effective and minimally invasive and should be considered actively for the treatment of symptomatic diaphragmatic eventration even in neonates.
\end{abstract}

Keywords: diaphragmatic eventration, thoracoscopic plication, neonate, single-lung ventilation

\section{Introduction}

Diaphragmatic eventration is caused by a congenital structural defect of the diaphragm or as a consequence of phrenic nerve injury. ${ }^{1)}$ Injury may be related to traction on

${ }^{1}$ Department of Pediatric General and Urogenital Surgery, Juntendo University School of Medicine, Tokyo, Japan

${ }^{2}$ Department of Anesthesiology, Juntendo University School of Medicine, Tokyo, Japan

Received: January 31, 2012; Accepted: April 3, 2012

Corresponding author: Tsubasa Takahashi, MD. Department of Pediatric General and Urogenital Surgery, Juntendo University School of Medicine, 2-1-1 Hongo, Bunkyo-ku, Tokyo 113-8421, Japan

Email: tsubasa@juntendo.ac.jp

(C)2012 The Editorial Committee of Annals of Thoracic and

Cardiovascular Surgery. All rights reserved. the nerve during birth or may be iatrogenic, occurring, for example, during open cardiac surgery. In infants and children who have diaphragmatic eventration, clinical manifestations are diverse, ranging from mild gastrointestinal symptoms to life-threatening respiratory distress requiring mechanical ventilatory support. ${ }^{2)}$ While usually asymptomatic, diaphragmatic eventration can cause progressive dyspnea on exertion or respiratory infection in small children, especially in those less than 2 years of age. ${ }^{3)}$

Traditionally, surgical intervention was complicated, particularly in symptomatic smaller children, but advancements in endoscopic surgery have allowed diaphragmatic eventration to be treated even in infants using thoracoscopy. The benefits of endoscopic surgery are evident in the literature and include smaller incision(s), quicker recovery, decreased pain, and specific treatment 


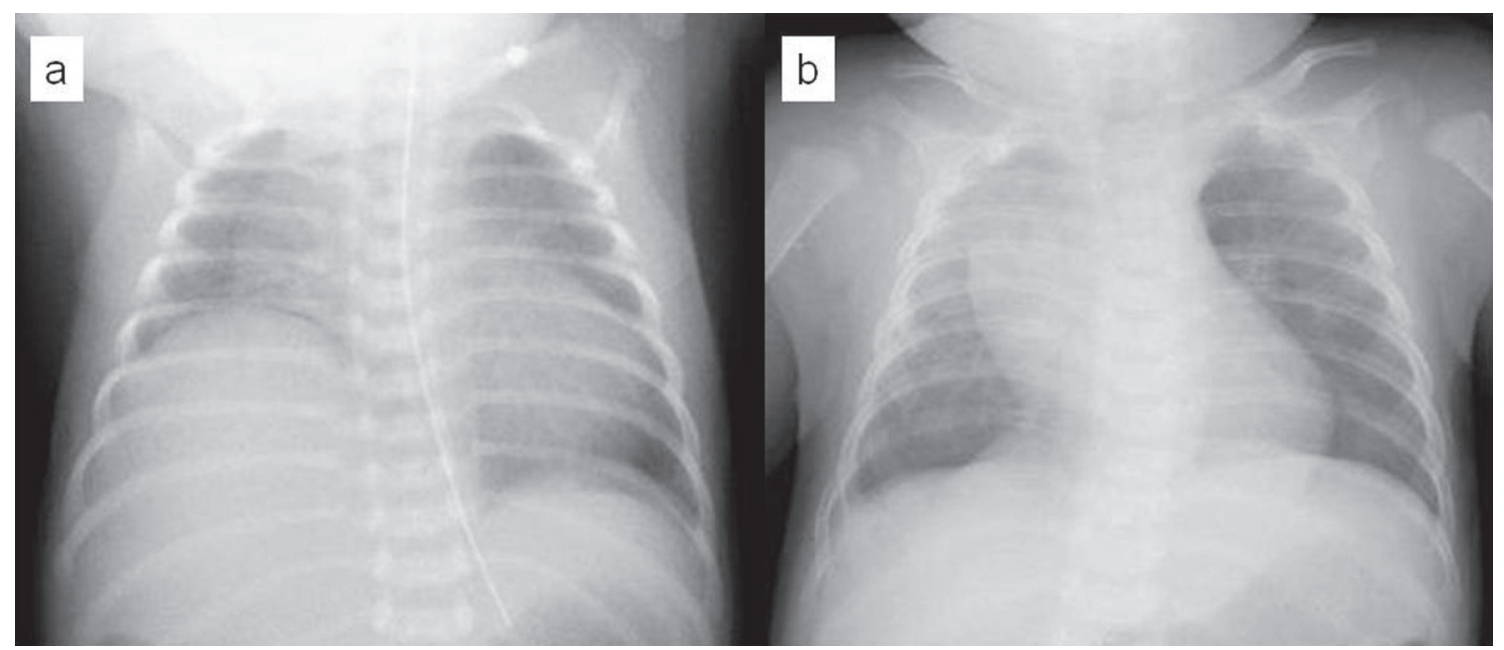

Fig. 1 a) Preoperative chest radiograph shows elevation of the right hemidiaphragm. b) Postoperative chest radiograph shows dramatic improvement.

of the exact problem because thoracoscopy is a more precise procedure. ${ }^{4)}$ Although thoracoscopic surgery is replacing thoracotomy in the pediatric age group, there are few reports describing thoracoscopic plication (TP) for diaphragmatic eventration in children, particularly in neonates. ${ }^{3,5,6)}$

Here, we report a case of TP performed using singlelung ventilation in a neonate with diaphragmatic eventration.

\section{Case Report}

A 10-day-old boy was referred for surgical management of right diaphragmatic eventration. Birth was at term, following an uncomplicated pregnancy and delivery. Shortness of breath, labored respiration and chest retraction presented soon after birth, necessitating mechanical ventilation. Chest radiography and computed tomography revealed an elevated right hemidiaphragm (Fig. 1a). Attempted weaning off mechanical ventilation failed with persistence of respiratory symptoms, requiring nasal directional positive airway pressure (DPAP). However, because there was no resolution of symptoms, TP was performed using a three port technique under single-lung ventilation on day 17 of life.

\section{Surgical procedure}

TP was performed under general anesthesia using single-lung ventilation with a Fogarty embolectomy catheter. The patient was placed in the left lateral decubitus position with his right arm raised to the level of his head in order to expose the chest fully. An optical $5 \mathrm{~mm}$ port was placed in the 5th intercostal space in the midaxillary line using an open technique. Other 5-mm ports were placed in the 5th intercostal space below the tip of the scapula and in the 5th intercostal space in the mammary line. Pneumothorax was induced, and carbon dioxide $\left(\mathrm{CO}_{2}\right)$ was insufflated at $4 \mathrm{mmHg}$ through the first port. Leftsided single-lung ventilation was achieved by blocking the right main bronchus with the Fogarty catheter. The bulging right hemidiaphragm could easily be seen, and surgery was facilitated (Fig. 2a). After inspection of the diaphragm, two trial pleats were prepared and approximated so that the degree of plication could be determined and the slack of the diaphragm positioned under the suture line. Plication of the diaphragm was commenced as far posterolaterally as possible and proceeded anteromedially using 10 interrupted 3-0 nonabsorbable sutures placed in extracorporeally. Adequate flattening of the hemidiaphragm was achieved (Fig. 2b). Trial decompression of the pneumothorax indicated that the diaphragm was tight, and the lung was re-expanded. Instruments were removed, and ports closed conventionally. No chest tube was placed.

The postoperative course was excellent with complete resolution of respiratory symptoms and no recurrence for 9 months to date. Chest radiography just 3 months postoperatively showed dramatic improvement (Fig. 1b).

\section{Discussion}

To the best of our knowledge, this is the first reported 


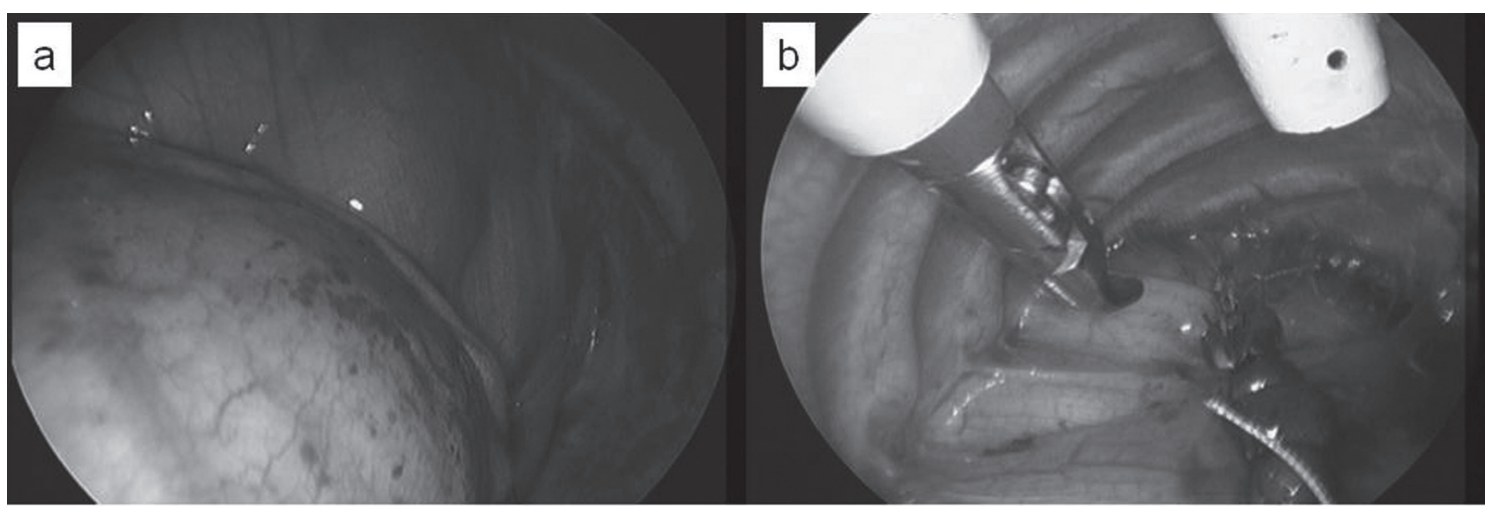

Fig. 2 a) Thoracoscopic view of the bulging thinned hemidiaphragm. b) Thoracoscopic view of the completed procedure.

case of thoracoscopic plication performed using singlelung ventilation for diaphragmatic eventration in a neonate.

The indications for and timing of plication for congenital diaphragmatic eventration are controversial. ${ }^{2}$ However, as the lungs grow until at least the age of 10 years, ${ }^{7)}$ it seems reasonable to provide space for future pulmonary development by plication without delay. Moreover, results with thoracoscopy are excellent with dramatic resolution in symptoms in infants and children. ${ }^{3,5,6)}$ Our case demonstrates that TP is feasible and can be performed safely even in a neonate; thus, TP should be actively considered as the treatment of choice for symptomatic diaphragmatic eventration in all age groups.

An issue of concern related to thoracoscopy in small children is the effects of $\mathrm{CO}_{2}$ insufflation. $\mathrm{CO}_{2}$ insufflation could lead to hypoxia, hypercapnea, hemodynamic instability, and acidosis ${ }^{8,9}$ because the permeability of $\mathrm{CO}_{2}$ is high in small children. ${ }^{910)}$ To minimize such risks, lower insufflation pressure $(<5 \mathrm{mmHg})$ has been reported, ${ }^{6}$ and we used single-lung ventilation and $4 \mathrm{~mm}$ $\mathrm{Hg}$ insufflation pressure without complications. In fact, single-lung ventilation allowed excellent exposure despite lower insufflation pressure.

There are reports in the literature that single-lung ventilation can be complicated in smaller infants because of difficulties encountered when blocking the bronchus. ${ }^{6,8)}$ However, as we found, single-lung ventilation is desirable during thoracoscopic surgery. Single-lung ventilation has been reported in infants and children ${ }^{11,12}$ but we found a Fogarty embolectomy catheter to be excellent for achieving safe, effective single-lung ventilation.

Technically, plication of the diaphragm should be commenced as far posterolaterally as possible, because there is a natural suture line created and plication can progress smoothly. Abraham et al. ${ }^{13)}$ reported that the first suture should be placed in the middle of the dome of the diaphragm, and can be tied without excessive tension on the suture line provide that the diaphragm is held in place at the desired level with a fan-shaped retractor. However, to achieve this, an additional working port is needed. In our case, the number of possible ports was limited because of single-lung ventilation and the size of our patient, but 3 ports were more than adequate for a successful TP, and no lung retractor was required. We also chose to start plicating posterolaterally because the closer the suture line gets to the mediastinum, the thicker the diaphragm becomes, and there may be more tension on sutures around the center of the diaphragm with risk for suture breakdown because the diaphragm itself is weaker in smaller infants, especially in neonates.

Sato et al. ${ }^{6}$ recommended plicating the elevated diaphragm from anterolateral to posterolateral to prevent nerve injury. However, the phrenic nerve divides into branches on the diaphragm and these mainly run laterally anterior and posterior to the lateral leaf of the central tendon in an anteromedial to posterolateral direction, especially in the periphery. ${ }^{14)}$ Therefore, it would seem more logical, anatomically, to plicate in a direction, from posterolateral to anteromedial, to prevent nerve injury.

Most surgeons believe interrupted sutures break down less than running sutures do. ${ }^{13,15)}$ Although there are no technical data that support this belief, from reports in the literature, ${ }^{16,17)}$ another port would have been required if running sutures were used to confirm that the suture line was tight. For our case, we used interrupted sutures because they can be placed using 2 operative ports while plicating the diaphragm progressively. 
In conclusion, to the best of our knowledge, this is the youngest case of TP for diaphragmatic eventration performed using single-lung ventilation. TP is safe, effective and minimally invasive and should be considered actively for the treatment of symptomatic diaphragmatic eventration even in neonates.

\section{Disclosure Statement}

Tsubasa Takahashi and other co-authors have no conflict of interest.

\section{References}

1) Wayne ER, Campbell JB, Burrington JD, et al. Eventration of the diaphragm. J Pediatr Surg 1974; 9: 64351.

2) Yazici M, Karaca I, Arikan A, et al. Congenital eventration of the diaphragm in children: 25 years' experience in three pediatric surgery centers. Eur J Pediatr Surg 2003; 13: 298-301.

3) Hines MH. Video-assisted diaphragm plication in children. Ann Thorac Surg 2003; 76: 234-6.

4) Georgeson KE, Robertson DJ. Minimally invasive surgery in the neonate: review of current evidence. Semin Perinatol 2004; 28: 212-20.

5) Becmeur F, Talon I, Schaarschmidt K, et al. Thoracoscopic diaphragmatic eventration repair in children: about 10 cases. J Pediatr Surg 2005; 40: 1712-5.

6) Sato M, Hamada Y, Takada K, et al. Thoracoscopic diaphragmatic procedures under artificial pneumothorax. Pediatr Surg Int 2005; 21: 34-8.
7) Inselman LS, Mellins RB. Growth and development of the lung. J Pediatr 1981; 98: 1-15.

8) Tanaka Y, Iwanaka T. Thoracoscopic diaphragmatic plication in small infants. Asian J Endosc Surg 2010; 3: 169-73.

9) Kalfa N, Allal H, Raux O, et al. Multicentric assessment of the safety of neonatal videosurgery. Surg Endosc 2007; 21: 303-8.

10) McHoney M, Corizia L, Eaton S, et al. Carbon dioxide elimination during laparoscopy in children is age dependent. J Pediatr Surg 2003; 38: 105-10; discussion 105-10.

11) Hammer GB. Single-lung ventilation in infants and children. Pediatr Anesth 2004; 14: 98-102.

12) Miyaji $K, \mathrm{Ka} K$, Okamoto $H$, et al. One-lung ventilation for video-assisted thoracoscopic interruption of patent ductus arteriosus. Surg Today 2004; 34: 1006-9.

13) Abraham MK, Menon SS, S BP. Thoracoscopic repair of eventration of diaphragm. Indian Pediatr 2003; 40: 1088-9.

14) Shah PL. Diaphragm and phrenic nerve. In: Standring S eds.; Gray's anatomy: The anatomical basis of clinical practice, 40th edn. Spain, Elsevier, 2008; pp 100712.

15) Kim do H, Joo Hwang J, Kim KD. Thoracoscopic diaphragmatic plication using three $5 \mathrm{~mm}$ ports. Interact Cardiovasc Thorac Surg 2007; 6: 280-1.

16) Hwang Z, Shin JS, Cho YH, et al. A simple technique for the thoracoscopic plication of the diaphragm. Chest 2003; 124: 376-8.

17) Arca MJ, Barnhart DC, Lelli JL, et al. Early experience with minimally invasive repair of congenital diaphragmatic hernias: results and lessons learned. J Pediatr Surg 2003; 38: 1563-8. 